\title{
Tips for Embryo Transfer Procedure in IVF/ICSI Cycle
}

\author{
Howaida Hashim ${ }^{1,2,3,4,5 *}$ \\ ${ }^{1}$ King Abdulaziz University, Saudi Arabia \\ ${ }^{2}$ Senior Embryologist, Saudi Arabia \\ ${ }^{3}$ Middle East Fertility Society Embryologist Interest Group, Saudi Arabia \\ ${ }^{4}$ IVF Laboratory, Saudi Arabia \\ ${ }^{5}$ Dr Erfan \& Bagedo General Hospital, Saudi Arabia
}

KEYWORDS: Embryo transfer; Pregnancy; IVF; ICSI; Embryo transfer procedure; Embryo transfer catheter; Difficult ET

\section{OPINION}

It is well known that the success of IVF/ICSI treatment cycle depends on many factors [1]. These factors could be related to patients treated (Age, BMI, Hormone levels, previous medical history, infertility duration, etiology and more), stimulation cycles (Stimulation protocol, duration of stimulation, drug used ... etc.), and laboratory process (quality of gametes, gamete handling, insemination technique (IVF/ICSI), embryology managing and incubation) $[2,3]$. In addition to an important factor, that is often not realized that is the embryo transfer procedure itself. The recommendation of the evidence-based guidelines for embryo transfer procedure is to avoid difficult embryo transfer which might be responsible for a failed implantation [4,5]. A variety of techniques are available, and many supporting devices are sometimes used during the procedure (for example; Wallace stylet (Malleable Stylet, Ref. 1816NST, Smiths), forceps, tenaculum, uterine sound etc.) for what they grade as a "difficult transfer" $[6,7]$. In other situations, the outer-sheath is inserted to assist in helping the introduction through the internal os, or a soft inner catheter that contained the embryos is inserted directly (e.g. Wallace catheter (Sure-Pro Ultra ${ }^{\circledR}$ Embryo Transfer Replacement Catheter, Ref. PE623, Wallace), Labotect (Embryo Transfer Catheter Set, Ref. 13366, Labotect $\mathrm{GmbH}$, Labor-Technik-Gottingen Kampweg 12,37124 Rosdorf, Germany) and others) [8]. It has been shown in the literature that symptoms (such as: bleeding, tenderness, contractions etc.) may play a role in pregnancy outcome and influence the interpretation of how difficult or easy the embryo transfer is [9]. These symptoms or signs occur during or after the embryo transfer and might interfere with embryo implantation. It was found that the uterine contractions might happen more on day- 3 than day- 5 due to the influence of progesterone [10-12].

In consideration of the above-mentioned points, few tips might be helpful for a more successful embryo transfer ending with a higher pregnancy rate.

1. Any mechanical measurement of the uterine cavity length is to be done as a mock on the day of egg retrieval after the procedure.

2. Using ultrasound guidance for embryo transfer appears to be combined with higher pregnancy rate $[13,14]$.

3. Try to determine the expected position for - softly and gentlydepositing (not push or put) the embryo.

4. Avoid touching the fundus.

If using the outer-sheath alone, there is a possibility of little uterine substances such as (blood, mucus, debris...etc.) is introduced into the outer sheath by capillarity, then, when inserting the inner catheter, the uterine substances might hinder the embryo(s) from
Quick Response Code:

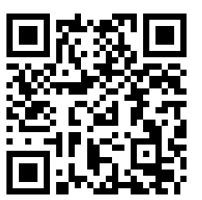

Address for correspondence: Howaida Hashim, Assistant Professor, Senior Embryologist, King Abdulaziz University, Saudi Arabia

Received: November 07, 2019 Published: November 12, 2019

How to cite this article: Howaida H. Tips for Embryo Transfer Procedure in IVF/ICSI Cycle. 2019 - 1(3) OAJBS.ID.000112. DOI: 10.38125/OAJBS.000112 
attaching the endometrial lining. To avoid this, insert the outer sheath together with the inner sheath but keep it hidden inside the outer sheath until you pass the internal os, hold the outer sheath and introduce the inner catheter with the embryo(s) to the determined location for depositing the embryo(s).

5. Retransferring the embryo(s) appears not to affect the pregnancy rate [15].

6. If an atraumatic transfer is not possible, the primary use should be the stylet before any external guidance may have to be considered.

7. Allowing the patient to watch the embryo transfer procedure on U/S monitor might restore her confidence, according to the literature this might increase her pregnancy chance.

\section{REFERENCES}

1. Cohen J (1989) Embryo replacement technology. San Francisco 31 Annual post graduate course. ASRM.

2. Mains L, Van Voorhis BJ (2010) Optimizing the technique of embryo transfer. Fertil Steril 94(3): 785-790.

3. Kovacs GT (1999) What factors are important for successful embryo transfer after in-vitro fertilization? Hum Reprod 14(3): 590-592.

4. Zegers-Hochschild F, Adamson GD, de Mouzon J, Ishihara O, Mansour $\mathrm{R}$, et al. (2009) The International Committee for Monitoring Assisted Reproductive Technology (ICMART) and the World Health Organization (WHO) Revised Glossary on ART Terminology. Hum Reprod 24(11): 2683-2687.

5. Spandorfer SD, Goldstein J, Navarro J, Veeck L, Davis OK, et al. (2003) Difficult embryo transfer has a negative impact on the outcome of in vitro fertilization. Fertil Steril 79(3): 654-655.
6. Lass A, Abusheikha N, Brinsden P (1999) The effect of a difficult embryo transfer on the outcome of IVF. Hum Reprod 14(9): 2417.

7. Lesny P, Killick SR, Robinson J, Raven G, Maguisness SD (1999) Junctional zone contractions and embryo transfer: is it safe to use a tenaculum? Hum Reprod 14(9): 2367-2370.

8. Frydman R (2004) Impact of embryo transfer techniques on implantation rates. J Gynecol Obstet Biol Reprod (Paris). 33(1Pt2): S36-39.

9. Saito H, Sato F, Hirayama T, Saito T, Kawagoe S, et al. (1992) Factors promoting embryo implantation in in vitro fertilization and embryo transfer. Horm Res 37(1): 64-68.

10. Fanchin R, Ayoubi JM, Righini C, Olivennes F, Schönauer LM, et al. (2001) Uterine contractility decreases at the time of blastocyst transfer. Hum Reprod 16(6): 1115-1119.

11.Zech NH, Lejeune B, Puissant F, Vanderzwalmen S, Zech H, et al. (2007) Prospective evaluation of the optimal time for selecting a single embryo for transfer: day 3 versus day 5. Fertil Steril 88(1): 244-246.

12. Fanchin R, Righini C, Olivennes F, Taylor S, Ziegler D, et al. (1998) Uterine contractions at the time of embryo transfer alter pregnancy rates after in-vitro fertilization. Hum Reprod 13(7): 1968-1974.

13. Drakeley A, Jorgensen A, Sklavounos J, Aust T, Gazvani R, et al. (2008) A randomized controlled clinical trial of 2295 ultrasound-guided embryo transfers. Hum Reprod 23: 1101-1106.

14. Biervliet FP, Lesny P, Maguiness SD, Killick SR (2002) Ultrasound-guided embryo transfer maximizes the IVF results on day 3 and day 4 embryo transfer but has no impact on day 5. Hum Reprod 17(4): 1131-1132.

15. Tur-Kaspa I, Yuval Y, Bider D, Levron J, Shulman A, et al. (1998) Difficult or repeated sequential embryo transfer do not adversely affect in-vitro fertilization pregnancy rates or outcome. Hum Reprod. 13(9): 24522455 . 